University of Nebraska - Lincoln

DigitalCommons@University of Nebraska - Lincoln

October 1992

\title{
Stars Classified as Constant in the General Catalog of Variable Stars
}

\author{
Edward G. Schmidt \\ University of Nebraska-Lincoln, eschmidt1@unl.edu \\ John R. Chab \\ University of Nebraska - Lincoln \\ Darwin E. Reiswig \\ University of Nebraska - Lincoln
}

Follow this and additional works at: https://digitalcommons.unl.edu/physicsschmidt

Part of the Physics Commons

Schmidt, Edward G.; Chab, John R.; and Reiswig, Darwin E., "Stars Classified as Constant in the General Catalog of Variable Stars" (1992). Edward Schmidt Publications. 21.

https://digitalcommons.unl.edu/physicsschmidt/21

This Article is brought to you for free and open access by the Research Papers in Physics and Astronomy at DigitalCommons@University of Nebraska - Lincoln. It has been accepted for inclusion in Edward Schmidt Publications by an authorized administrator of DigitalCommons@University of Nebraska - Lincoln. 


\title{
Stars Classified as Constant in the General Catalog of Variable Stars
}

\author{
EDWARD G. SCHMIDT, John R. ChaB, AND DaRwIN E. ReISWIG \\ Behlen Observatory, Department of Physics and Astronomy, University of Nebraska-Lincoln, Lincoln, Nebraska 68588-0111 \\ Electronic mail: eschmidt@unlinfo.unl.edu \\ Received 1992 April 13; accepted 1992 July 2
}

\begin{abstract}
Photometric observations have been made of 16 stars classified as constant, CST or CST:, in the General Catalog of Variable Stars. One star, U Tauri, was found to be a rapid irregular variable and three others may vary at a low level. In two cases, FV Delphini and FP Geminorum, the star identified as the variable on published finding charts is not variable but a nearby star did vary. It is suggested that the wrong stars were marked on the charts but the possibility remains that the variables may have exhibited constancy during earlier observations.
\end{abstract}

\section{INTRODUCTION}

The fourth edition of the General Catalog of Variable Stars (the GCVS, Kholopov 1985, 1987) contains 69 stars which are classed as constant in light (CST) and 89 stars which are classed as constant with some uncertainty (CST:). The introduction to the catalog characterizes these as "nonvariable stars, formerly suspected to be variable and hastily designated. Further observations have not confirmed their variability."

While many of these stars were no doubt erroneously included in the catalog, Eggen (1983) found that such one star, R Puppis, began to vary in 1980 after decades of apparent constancy. Since the star had been reported as a high amplitude variable a century earlier, it is apparent that $R$ Pup is a variable which went through a long interval of inactivity. A similar case is presented by RU Camelopardalis which pulsated with high amplitude until about 1964 when Demers and Fernie (1966) found it to be constant. Since that time pulsation has been reestablished but not with the regularity the star exhibited previously (see Broglia et al. 1978). These stars suggest that pulsation can disappear for periods of years or decades and then resume.

The cessation of variability in R Pup and RU Cam is of interest because it demonstrates significant changes in stellar pulsation on time scales of years or decades. This is short compared with the time scales in most stages of stellar evolution but long compared with the pulsation period. It is, however, comparable to the time scale associated with such phenomena as the solar cycle and the variation in the strength of the Blazhko effect. The Kelvin time can also be of this order for luminous stars.

In view of the potential interest in mechanisms which might produce pauses in stellar variation on time scales of years or decades, an attempt has been made to locate more examples. Such stars might be entered in the GCVS after being observed during an active phase but later deemed constant when reobserved during a quiescent phase. We have therefore reobserved 16 stars classed as CST or CST: in the GCVS.

\section{THE OBSERVATIONS}

The observations presented in this paper were made between 1991 September and 1992 March with the Behlen Observatory CCD photometry system. The observational techniques were described previously (Schmidt et al. 1990) as was the instrumentation (Schmidt 1988, 1990, 1991). As in the previous papers, observations were made in the $V$ and $R$ bands. It should be noted that differential magnitudes relative to other stars in the CCD field are determined accurately even when conditions are too poor for all sky photometry. For the present study we shall be primarily concerned with the differential magnitudes although data were placed on the standard system using photometric nights.

The stars are listed in Table 1. The first column identifies them while the second and third columns give the number of nights on which each was observed and the number of individual observations. The fourth and fifth columns give the night-to-night rms scatter of the $V$ and $R$ differential magnitudes. $V$ magnitudes and $V-R$ colors on the standard system (as defined by the standard stars of Landolt 1983) are listed in columns 6 and 8 while their standard errors are in columns 7 and 9.

The final column of Table 1 indicates whether each star was variable during the interval of our observations. This judgment was based on the standard deviations listed in the table, the number and brightnesses of the comparison stars, and any systematic trends in the magnitudes during individual nights. Thus, VV Cam is listed as a possible variable in spite of small night-to-night scatter in its $V$ and $R$ magnitudes.

\section{DISCUSSION OF INDIVIDUAL STARS}

Stars which are identified in Table 1 as possible variables will not be considered further here. However, since some of these objects may be in the early stages of reestablishing variability, they will be observed again in future seasons.

The three definite variable stars are listed in Table 2 with their identifications and coordinates from the Hubble Space Telescope Guide Star Catalog (hereafter referred to 
TABLE 1

Data for Stars Classified as Constant in the GCVS

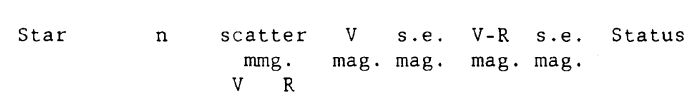

\begin{tabular}{lrrrrrrrrr}
\hline TY Aur & 16 & 16 & 10 & 10 & 10.64 & 0.01 & 0.59 & 0.01 & $\mathrm{a}$ \\
V2 Aur & 13 & 27 & 34 & 17 & 10.99 & 0.01 & 0.20 & 0.01 & $\mathrm{~b}$ \\
AV Aur & 10 & 14 & 27 & 34 & 9.64 & 0.02 & 0.98 & 0.01 & $\mathrm{~b}$ \\
VV Cam & 9 & 18 & 13 & 14 & 12.77 & 0.01 & 0.48 & 0.01 & $\mathrm{~b}$ \\
VW Cam & 17 & 18 & 29 & 22 & 12.93 & 0.01 & 0.58 & 0.01 & $\mathrm{a}$ \\
RV Cnc & 7 & 8 & 9 & 8 & 12.78 & 0.01 & 0.31 & 0.01 & $\mathrm{a}$ \\
FV Del & 18 & 18 & 121 & 82 & 12.15 & -- & 1.55 & -- & $\mathrm{c}$ \\
Z Gem & 5 & 8 & 9 & 10 & 12.34 & 0.01 & 0.70 & 0.01 & $\mathrm{a}$ \\
TT Gem & 6 & 6 & 9 & 10 & 12.6 & -- & 0.9 & -- & $\mathrm{a}, \mathrm{d}$ \\
WX Gem & 5 & 5 & 13 & 14 & 10.29 & 0.01 & 0.21 & 0.02 & $\mathrm{a}$ \\
FL Gem & 12 & 12 & 17 & 33 & 14.34 & 0.02 & 0.39 & 0.01 & $\mathrm{a}$ \\
FP Gem & 11 & 14 & 137 & 97 & 9.6 & -- & 1.3 & -- & $\mathrm{e}$ \\
BQ Per & 7 & 7 & 16 & 7 & 12.16 & 0.01 & 0.40 & 0.01 & $\mathrm{a}$ \\
KO Per & 7 & 9 & 9 & 17 & 12.96 & 0.02 & 0.83 & 0.02 & $\mathrm{a}$ \\
U Tau & 29 & 55 & 133 & 135 & 10.3 & -- & 0.35 & -- & $\mathrm{f}$ \\
RT Tau & 7 & 13 & 13 & 17 & 9.90 & 0.02 & 0.17 & 0.02 & $\mathrm{a}$ \\
& & & & & & & & & \\
\hline
\end{tabular}

a Constant

Possible variable

c The star identified on the published finding chart as FV Del is constant. The data given here refer to the nearby variable.

d TT Gem was only observed on one photometric night. Hence, an approximate magnitude and color are given.

e The star identified on the published finding chart as

FP Gem is constant. The data given here refer to the nearby variable.

f Variable

as GSC; described by Lasker et al. 1990; Russell et al. 1990; and Jenkner et al. 1990). They will now be discussed individually.

\subsection{FV Del}

FV Del was observed over an interval of 86 days. The star identified on the published finding chart (MVS 1957) was found to be constant in light. However, a star 9" S and $46^{\prime \prime} \mathrm{E}$ of that object varied over a range of $0.46 \mathrm{mag}$ during the interval of the observations. There is no other variable listed in the GCVS that close to FV Del. It seems likely that the finding chart was marked erroneously and the nearby star is actually FV Del. This also suggests that Bond's (1978) spectral classification of G0 does not refer to the variable.

The $V$ magnitudes and $V-R$ colors for FV Del are plotted against Heliocentric Julian Date in Fig. 1. It can be seen that the star went through 2 unequal maxima which are separated by about 55 days. The minima are also unequal in depth although the star had not reached the bot-

TABLE 2

Stars Which Were Found to Vary

\begin{tabular}{|c|c|c|c|}
\hline $\begin{array}{c}\text { Variable } \\
\text { Star } \\
\text { Name }\end{array}$ & $\begin{array}{c}\text { HST GSC } \\
\text { Designation }\end{array}$ & $\begin{array}{l}\text { R, A. } \\
\text { (epoch }\end{array}$ & $\begin{array}{l}\text { Dec. } \\
2000 .)\end{array}$ \\
\hline FV De 1 & GSC1086 2036 & $20: 21: 46.3$ & $14: 07: 49$ \\
\hline FP Gem & GSC1331 1038 & $6: 55: 37.2$ & $15: 35: 03$ \\
\hline U Tau & GSC1272 0656 & $4: 21: 50.1$ & $19: 48: 51$ \\
\hline
\end{tabular}
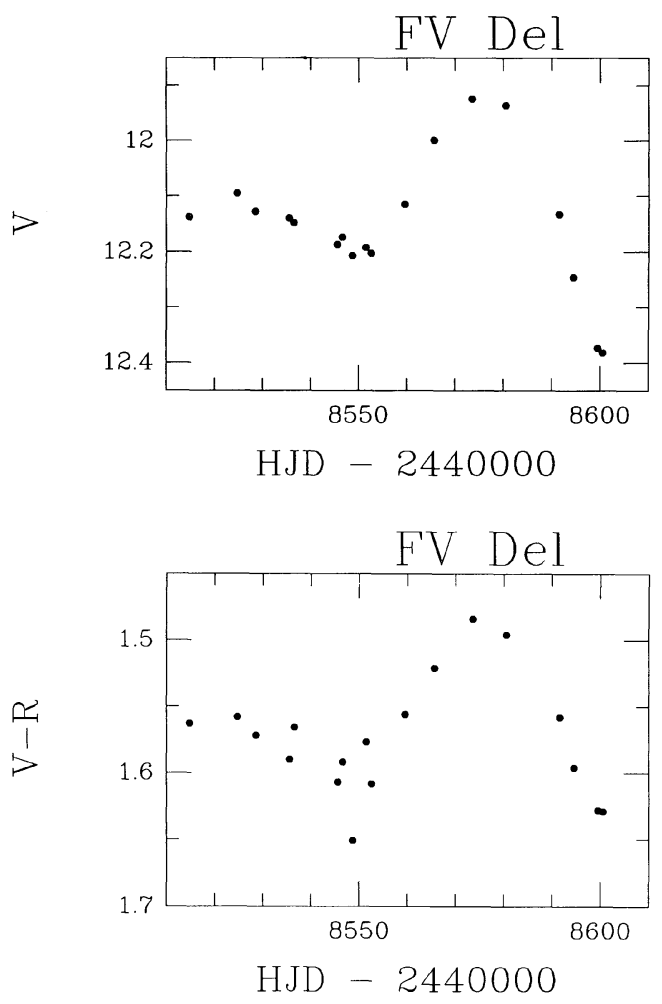

FIG. 1-The $V$ magnitude and $V-R$ color of FV Del plotted against the Heliocentric Julian Date.

tom of the second minimum at the end of the series of observations. The colors are well correlated with the magnitudes as should be the case for a pulsating variable. This behavior and the time scale suggest that FV Del is an RV Tauri star.

FV Del is classified as a long period variable or possibly an irregular variable in the GCVS, 3rd ed. (Kukarkin et al. 1969). This is the only edition of the GCVS to regard it as variable. Given the quantity and quality of data then available, this is probably not inconsistent with our conclusion.

RV Tau stars commonly exhibit excess emission in the infrared (Gehrz 1972). A source is present in the IRAS catalog (IRAS 1988) which matches the coordinates of FV Del. Following Deasy and Butler (1986) we find that $V$ - [12], the color index formed from our $V$ magnitude and the $12 \mu \mathrm{m} I R A S$ flux, is 10.8 . Spectral types for RV Tau stars can range from $F$ to $M$ (Gehrz 1972) which with our $(V-R)$ color gives a range from 0.6 to 1.3 magnitudes for $E(V-R)$ or a range from about 3.8 to about 8.0 mag for the visual absorption. Applying this we get a dereddened $V-$ [12] between 2.8 and 7. For classical Cepheids without infrared excesses and nonvariable supergiants, $V-[12]$ ranges from about 5 to 6.5 . Thus, the uncertainty in reddening for FV Del prevents us from drawing any conclusion regarding an infrared excess. This situation would be alleviated if a spectral type were known for the correct star.

The classification of FV Del as CST: in the GCVS is based on Meinunger's (1980) photographic photometry from the Sonneberg plate collection. While the misidenti- 

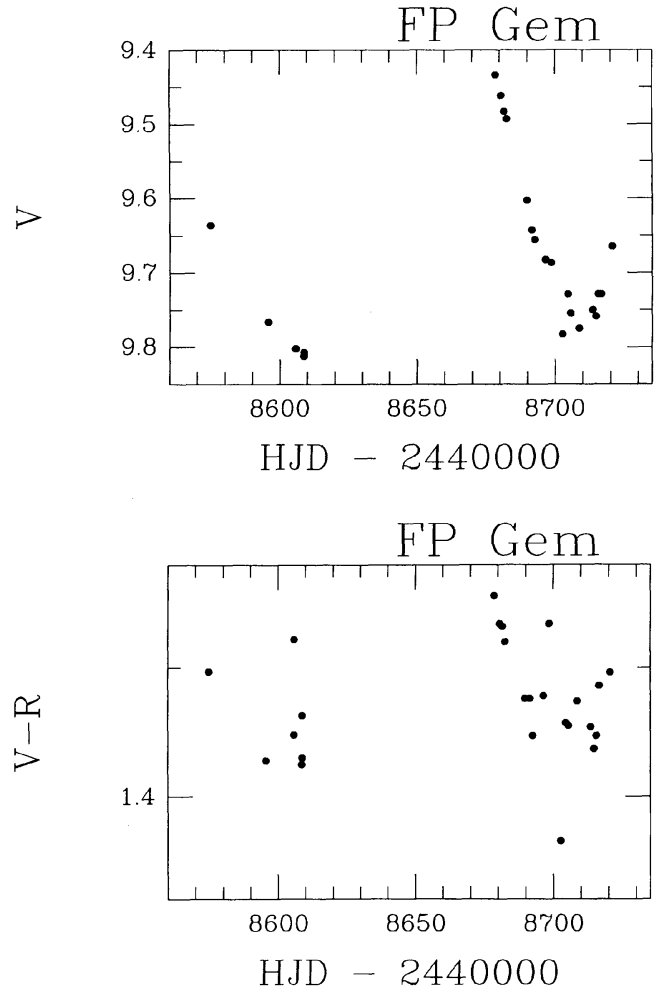

FIG. 2-The $V$ magnitude and $V-R$ color of FP Gem plotted against the Heliocentric Julian Date.

fication on the published finding chart may account for the failure to detect variation, it seems strange that large variation in a star less than an arcminute away would pass unnoticed. Thus, the possibility remains that FV Del has gone through episodes of variation and through episodes of constancy. Clearly, this star should be observed in the future to monitor its long-term behavior.

\subsection{FP Gem}

The case of FP Gem is similar to that of FV Del. Again the star marked on the published finding chart (Kurochkin 1948 ) is constant but a star 54" $\mathrm{N}$ and 54" E of the marked star exhibits variation. However, shortly after its discovery, Hoffmeister et al. (1954) gave a photographic magnitude for FP Gem which ranged from 13.2 to 13.8. This is much fainter than the star we find to vary but consistent with the star identified on the finding chart. We cannot resolve this confusion and will refer here to the star we find to vary as FP Gem.

We present the light and color curves in Fig. 2. Unfortunately, our coverage has a large gap. The slope of the decline at the end of our series of observations is appropriate to either a classical Cepheid with a period between 35 and 85 days or a Mira (Schmidt and Wiese 1989). An RV Tau star is another possibility. We cannot distinguish among these alternatives due to the gap in our data. Unfortunately, no spectral type is available to help decide this point and the GCVS, 2nd ed. (Kukarkin et al. 1958, the last edition to consider FP Gem to be variable) only classes it as an irregular variable (RW Aurigae type).

Hoffmeister et al. (1954) remark that FP Gem has a strongly variable amplitude but were able to derive a fiveday period. In view of the confusion regarding the identification, it is unclear how to interpret the conflict with our data. It is very improbable that the star marked on the finding chart stopped varying while our star began to vary sometime in the last 40 years. On the other hand, the fiveday period and faint magnitude given by Hoffmeister et al. are inconsistent with our observations. Whatever the case, there seem to have been significant changes in the pulsation of FP Gem over this time.

As was the case for FV Del, there is an IRAS point source which matches the location of FP Gem. Again the lack of a spectral type prevents us from determining whether there is an infrared excess in $V-$ [12]. However, unlike FV Del, this star was also measured at $25 \mu \mathrm{m}$ by $I R A S$. The ratio of $F[25] / F[12]=0.3$ is in accord for values for nonvariable supergiants and Cepheids with no infrared excess (see Fig. 1 of Deasy and Butler 1986). This argues for classifying FP Gem as a Cepheid.

The nonvariability for FP Gem was based on observations by Meinunger (1966). As for FV Del, the misidentification of the variable could account for the lack of variation reported by that investigator but we wonder why the variation of such a nearby star was missed. We again speculate that FP Gem may have undergone episodes of light constancy. It too will be reobserved in future seasons to assess its long-term behavior as well as to clarify the variable star type.

\subsection{U Tau}

The $V$ magnitudes and $V-R$ colors of $U$ Tau are plotted against Heliocentric Julian Date in Fig. 3. It can be seen that there is a range of about $0.7 \mathrm{mag}$ in $V$ and about $0.75 \mathrm{mag}$ in $V-R$. However, the variations were so rapid that no light curve form is visible. Although it is not apparent from the plot, the colors and the magnitudes for this star are uncorrelated.

After the rapidity of the variations became obvious, multiple observations were obtained on a number of nights. An attempt was made to determine a period using the data-compensated discrete Fourier transform method (Ferraz-Mello 1981). The strongest peak in the power spectrum corresponded to 0.46591 days. However, the light curve with this period exhibits considerable scatter suggesting that the star is irregular with a time scale of about half a day. The GCVS classifies such stars as IN or IS stars on the basis of the irregularity, the large amplitude, and the short time scale.

While the IN stars are associated with nebulosity, the IS stars are described in the introduction to the GCVS as "rapid irregular variables with no apparent connection with diffuse nebulae, showing light changes by 0.5 to 1.0 mag during several hours or days." No nebulosity can be seen in vicinity of $U$ Tau on the Palomar Sky Survey prints although there is a faint nebulosity 15 arcmin to the south. 

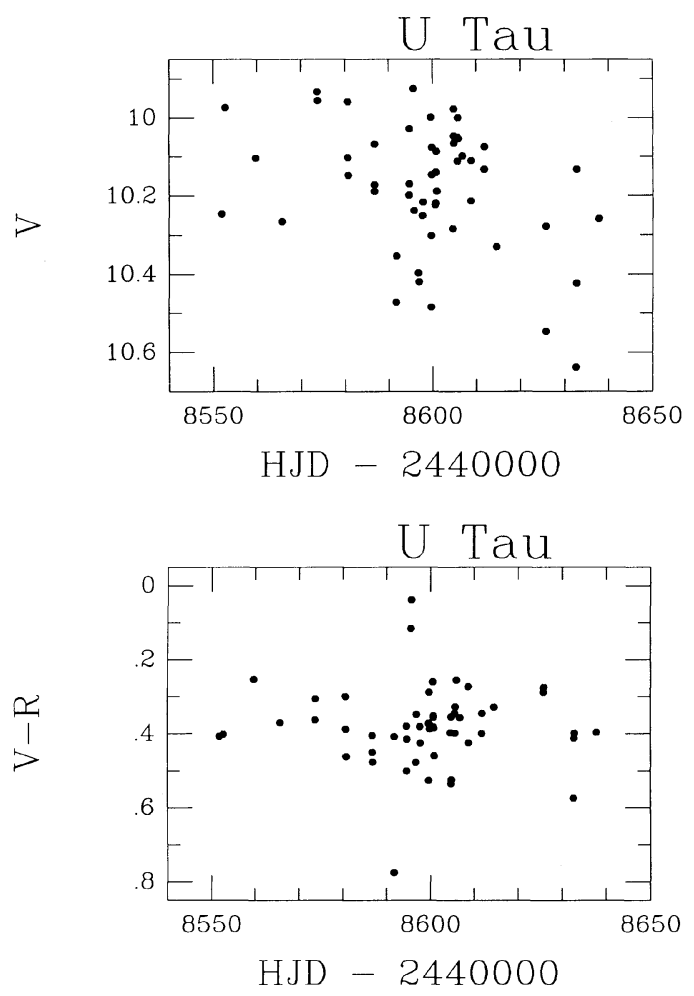

FIG. 3-The $V$ magnitude and $V-R$ color of $\mathrm{U}$ Tau plotted against the Heliocentric Julian Date.

The GCVS further divides the IN and IS stars into types $\mathrm{A}$ and $\mathrm{B}$ depending on whether the spectral type is earlier or later than F0. With an HD spectral type of F5, U Tau is thus of type ISB.

Baxendell (1862) first discovered the variability of $U$ Tau. He reported regular variation with an amplitude of more than a magnitude. However, Knott (1899) observed U Tau visually between 1863 and 1891 . His observations are plotted in Fig. 4. They are too widely scattered in time to show whether the star varied rapidly at that time as during the present set of observations. However, it is interesting that the range of Knott's observations appears to have decreased over the years. While the reduction in the range of the magnitude estimates may represent an artifact of the visual observations it should be noted that Knott's

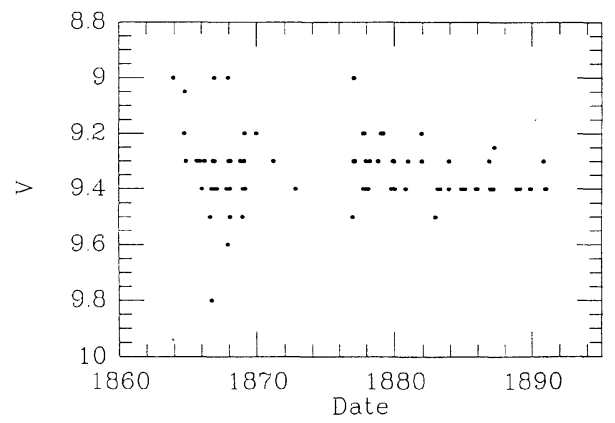

FIG. 4-V magnitude estimates for $U$ Tau by Knott plotted against the year. estimates for this star were based on a comparison with two stars of magnitudes 9.0 and 9.8. Thus, on four occasions prior to $1877 \mathrm{Knott}$ judged $\mathrm{U}$ Tau to equal the brighter of the comparison stars. However, he never found it to be that bright after 1877 . Thus, the apparent reduction in amplitude rests to a large degree on judging the equality of two stars rather than estimating where a star lies between two comparison stars. This circumstance argues for accepting the apparent amplitude reduction.

The present $V$ magnitudes range from 9.93 to 10.64 with a mean of about 10.2. On the other hand, Knott's magnitudes ranged from 9.0 to 9.6 (ignoring one marked as uncertain) early in his series of observations and converged to a value of 9.4 by the last 5 years. This discrepancy could easily be the result of a different magnitude scale in the observations of a century ago. Since we have not observed Knott's comparison stars, a direct check is not possible. However, we compared the comparison star magnitudes from Knott with the $V$ magnitudes from the GSC. For all 15 stars we found that Knott's magnitudes averaged $0.05 \mathrm{mag} \pm 0.59$ (standard deviation for one star) brighter than the GSC. If we restrict the comparison to the stars brighter than 11th magnitude, the difference is nearly the same, $0.07 \mathrm{mag}$, but the standard deviation drops to 0.28 . Finally, we note that Knott's adopted magnitudes for the two stars actually used for his U Tau estimates are 0.20 and 0.17 mag brighter than the GSC values. We selected 19 stars which were observed as comparison stars for various variables in the general region of the sky near Taurus. Our magnitudes for these stars averaged 0.27 mag \pm 0.21 fainter than the GSC magnitudes. Combining these two comparisons we conclude that differences in the magnitude scales might account for no more than half a magnitude of the discrepancy between our mean magnitude for U Tau and that of Knott. This seems to suggest that $\mathrm{U}$ Tau was at least several tenths of a magnitude brighter a century ago than it is now.

A further peculiarity is found in the GSC magnitude of $\mathrm{U}$ Tau, $V=8.91$. This is brighter than any of our magnitudes or those of Knott. Yet our value for the comparison star (GST1272 0406, 62" north of U Tau) differs by only 0.02 from the GSC value. Whether U Tau was actually more than a magnitude brighter a decade ago (when the GSC plate was obtained) depends critically on the reliability of the GSC magnitude. While the calibration sequences in most GSC fields do not extend to stars as bright as U Tau, by chance the sequence for the plate in question included a star with $V=8.38$. Thus, the brightness of $U$ Tau should not have produced errors at the level of a magnitude. Significant errors can occur near the edges of the plates (see Fig. 6 of Russell et al. 1990) and U Tau is close to the plate edge. However, the comparison star should be affected in a similar fashion and it would be surprising to find an error as great as a magnitude between the two. Nonetheless, about $1 \%$ of stars in plate overlap regions of the GSC were found by Russell et al. (1990) to show discrepancies $>1$ mag. Thus, we cannot be certain that $U$ Tau has faded by a magnitude over the past decade. How- 
ever, the above circumstances strongly suggest that this was the case.

While the comparison of the old observations with the recent ones involves considerable uncertainty, it seems safe to conclude that $\mathrm{U}$ Tau underwent an interval of quiescence but is now varying rapidly. Furthermore, there is evidence that $U$ Tau has changed significantly in mean magnitude over a period of years. This star should be observed in future seasons to determine the long-term nature of its variation.

This research was supported by Grant No. AST8815806 from the National Science Foundation. The reduction and data analysis were carried out using the facilities of the Minnich Astronomical Computing Center which was donated by Commander Charles B. Minnich. We thank Dr. E. P. Belserene for the use of her discrete data corrected Fourier period search program. This research made use of the Simbad database, operated at CDS, Strasbourg, France.

\section{REFERENCES}

Baxendell 1862, Manch. Proc., 3, 43

Broglia, P., Conconi, P., and Guerrero, G. 1978, A\&AS, 33, 339

Deasy, H., and Butler, C. J. 1986, Nature, 320, 726

Demers, S., and Fernie, J. D. 1966, ApJ, 144, 440

Eggen, O. J. 1983, AJ, 88, 386

Ferraz-Mello, S. 1981, AJ, 86, 619

Gehrz, R. D. 1972, ApJ, 178, 715

Hoffmeister, C., Ahnert-Rohlfs, E., Ahnert, P., Huth, H., and Gotz, W. 1954, Veroff. Sonneberg, 2, No. 2
IRAS 1988, IRAS Point Source Catalog (Washington, DC, NASA)

Jenkner, H., Lasker, B. M., Sturch, C. R., McLean, B. J., Shara, M. M., and Russell, J. L. 1990, AJ, 99, 2082

Kholopov, P. N. 1985, General Catalog of Variable Stars, 4th ed., Vols. 1 and 2 (Moscow, Nauka)

Kholopov, P. N. 1987, General Catalog of Variable Stars, 4th ed., Vol. 3 (Moscow, Nauka)

Knott, G. 1899, MemRAS, 52, 1

Kukarkin, B. V., Kholopov, P. N., Efremov, Yu. N., and Melvedeva, G. I. 1969, General Catalog of Variable Stars, 3rd ed. (Moscow, Sternberg State Astronomy Institute)

Kukarkin, B. V., Paranego, P. P., Efremov, Yu. N., and Kholopov, P. N. 1958, General Catalog of Variable Stars, 2nd ed. (Moscow, Sternberg State Astronomy Institute)

Kurochkin, N. E. 1948, Perem. Zvezd, 6, 94

Landolt, A. U. 1983, AJ, 88, 439

Lasker, B. M., Sturch, C. R., McLean, B. J., Russell, J. L., Jenkner, H., and Shara, M. M. 1990, AJ, 99, 2019

Meinunger, L. 1966, Mitt. uber veranderlichen Sterne, 3, 137

Meinunger, L. 1980, Veroff. Stern. Sonneberg, 3, 197

MVS, 1957, Mitt. uber veranderliche Sterne, 308

Russell, J. L., Lasker, B. M., McLean, B. J., Sturch, C. R., and Jenkner, H. 1990, AJ, 99, 2059

Schmidt, E. G. 1988, in Automated Small Telescopes, ed. D. S. Hayes and R. M. Genet (Mesa, Fairborn), p. 195

Schmidt, E. G., 1990, in Proceedings of the ASP Symp., Robotic Observatories, Boston, July 1990 (Mesa, Fairborn)

Schmidt, E. G., 1991, ASP Conf. Ser., Robotic Telescopes in the 1990s, ed. A. Filipenko (in press)

Schmidt, E. G., Loomis, C. G., Groebner, A. T., and Potter, C. T. 1990, ApJ, 360, 604

Schmidt, E. G., and Wiese, K. H. 1989, AJ, 98, 1659 